Bénédicte Abraham

Universität der Franche-Comté, Besançon

DOI: $10.19195 / 0435-5865.141 .2$

\title{
Die langsame Entstehung eines wissenschaftlichen Diskurses über Kunst und Literatur um 1800 im Licht des Briefwechsels zwischen Goethe und Schiller (1798-1805)
}

\section{Einleitung}

Ab $1794^{1}$ bis zum Tode Schillers im Jahre 1805 tauschten Goethe und Schiller regelmäßig Briefe aus. Mit dem frühzeitigen Tod Schillers wurde einer tiefen Freundschaft und einem fruchtbaren Arbeitsverhältnis ein Ende gesetzt. Die weit verbreitete Idee, die beiden Männer seien durch eine unzerstörbare Freundschaft verbunden gewesen, ist dennoch durch manche Kritiker revidiert worden. Richard Friedenthal vermutet zu Recht, dass die Beziehung zwischen Goethe und Schiller im Spannungsfeld zwischen Freundschaft und Rivalität anzusiedeln sei (1968: 441-442) ${ }^{2}$; Richard Friedenthal spricht sogar über eine Art Waffenstillstand zwischen zwei Großmächten, die über eine Demarkations- oder Grenzlinie hinaus Briefe austauschten (1968: 430). ${ }^{3}$ Folgende Darstellung hat einerseits der Freundschaft zwischen beiden Männern und andererseits ihren Reflexionen über ihre im Verschwinden begriffene Epoche und den Epochenübergang Rechnung zu tragen. Deshalb nehmen folgende Seiten den Übergang vom 18. zum 19. Jahrhundert in den Blick, den man hier im Licht des Briefwechsels zweier seiner prägenden

${ }^{1}$ Johann Wolfgang Goethe und Friedrich Schiller lernten sich im Juli 1794 nach einer Sitzung der «Naturforschenden Gesellschaft» in Jena kennen.

2 Richard Friedenthal, Goethe. Sein Leben und seine Zeit. Bd II, DTV, München 1968, S. 441442.

3 Ibidem., S. 430. Über die komplexe Freundschaft zwischen Goethe und Schiller berichtet auch Rüdiger Safranski in: Goethe und Schiller: Geschichte einer Freundschaft, Hanser, München 2009. 
Vertreter lesen möchte. Einleitend zur Analyse des Briefwechsels kann darauf hingewiesen werden, dass die Forschungsliteratur zu diesem Werk eher gering ist $^{4}$. Wie die Bibliographie ausweist, sind zum Briefwechsel zwischen Goethe und Schiller nur seltene Monographien erschienen. Und doch ist der aus diesen Briefen bestehende Korpus nicht nur hinsichtlich der manchmal ambivalenten Freundschaft zweier der größten Schriftsteller und Denker Deutschlands um 1800 interessant; folgende Analyse begründet sich auch durch den Inhalt der Briefe selbst, die sowohl von der bemerkenswerten Entwicklung der Philosophie (die Schriften Fichtes und Kants gehören z.B. zu den Lektüren Goethes) und den ästhetischen Theorien von $\mathrm{Kant}^{5}$ bis Hegel (Lukács 1955: 74-107), als auch von den wissenschaftlichen Fortschritten auf den Gebieten der Astronomie, der Elektrizität, der Naturwissenschaften, der Optik berichten, denen Goethe ein besonderes Interesse widmet. Bezieht man sich näher auf den Briefwechsel zwischen Goethe und Schiller, so stellt man fest, dass er für den Leser ein extrem reiches Dokument darstellt, das über eine Epochenschwelle ein neues Licht wirft. Als erste allgemeine Tendenz ist zu beobachten, dass die Briefe nicht nur viele prosaische Bemerkungen über das Wetter oder die Laune und den Gesundheitszustand ihrer jeweiligen Verfasser enthalten, sondern auch kritische Betrachtungen über die literarische, philosophische oder wissenschaftliche Entwicklung der Epoche entwerfen; in ihren markanten Punkten zeigen diese Briefe, wie Goethe und Schiller als besonders akute Seismografen ihrer Epoche zu verstehen sind und wie ihre Überlegungen im Kontext einer Zeit von bedeutenden Paradigmenwechseln stehen. Mithilfe der von uns ausgewählten Einteilung in verschiedenen Punkten lassen sich die Hauptaspekte des Briefwechsels aufzeigen und summarisch darstellen.

Neben dem ständigen Verweis auf die von ihnen geschaffenen Werke, enthalten ihre Briefe bald nachsichtige, bald strenge Betrachtungen über die jüngeren Publikationen oder wissenschaftlichen Experimente ihrer Zeit. Im Folgenden werden die verschiedenen Kommentare darüber immer durch repräsentative Zitate präzisiert. Die Wahrnehmung jener Briefe ganz auf ihren dokumentarischen Aspekt zu begrenzen, kann aber nicht ganz sinnvoll sein, insofern als in den Briefen das Fehlen von politischen Betrachtungen oder Analysen deutlich ist, wie Richard Friedenthal es betont hat (1968: 428) ${ }^{6}$; die Lektüre der Briefe lehrt eher, dass das Interesse der beiden Männer ausschließlich literarischen, ästhetischen und wissen-

${ }^{4}$ Der Briefwechsel zwischen Schiller und Goethe [hg. v. Bernhard Fischer und Norbert Oellers], Erich Schmidt Verlag, 2011; Zweiheit im Einklang: der Briefwechsel zwischen Schiller und Goethe; eine Ausstellung des Goethe-und Schiller-Archivs im Renaissancesaal der Herzogin Anna Amalia Bibliothek, 18.09.2009 bis 17.01.2010 [hg. v. Silke Henke und Alexander Rosenbaum], Klassik Stiftung Weimar, Weimar 2009.

5 Über die Bedeutung der Kantischen Philosophie in diesem Text kann man den Beitrag von Shu Ching Ho lesen: «Ausgleich der Gegensätze. Zur Bedeutung der Kantischen Philosophie im Briefwechsel zwischen Schiller und Goethe», in: Der Briefwechsel zwischen Schiller und Goethe [hg. v. Bernhard Fischer und Norbert Oellers], Erich Schmidt Verlag, Berlin 2011, S. 101-120.

${ }^{6}$ R. Friedenthal, op. cit., S. 428. 
schaftlichen Fragen gilt. Zu der Zeit, als Goethe und Schiller regelmäßig Briefe auszutauschen beginnen, arbeitet Schiller an seinem Drama Wallenstein (Lukács 1955: 76) und Goethe ist - zumindest in den Jahren zwischen 1794 und 1797 mit seinem Theaterstück Hermann und Dorothea sowie mit Faust beschäftigt; die Arbeit an der Tragödie Faust fällt ihm manchmal schwer, wie es aus einigen Briefen an Schiller hervorgeht: "Von Faust kann ich jetzt nichts mitteilen; ich wage nicht das Paket aufzuschnüren, das ihn gefangen hält. Ich könnte nicht abschreiben ohne auszuarbeiten, und dazu fühle ich mir keinen Mut.» schreibt er am 2. Dezember 1794. Drei Jahre später liest man folgende Anmerkung im Brief vom 17. August 1797: «Mit diesem letzten geht mir's wie mit einem Pulver, das sich aus seiner Auflösung nun einmal niedergesetzt hat; solange Sie dran rütteln, scheint es sich wieder zu vereinigen, sobald ich wieder für mich bin, setzt es sich nach und nach zu Boden.» (Goethe 1950: 97) Die ausgewählte Textstelle ist nicht nur exemplarisch für den traditionellen Inhalt des Briefwechsels, der hauptsächlich eine produktionsästhetische Dimension formuliert, sondern auch für die wissenschaftliche Metapher der Auflösung des Pulvers, auf die Goethe in dieser Textstelle zurückgreift. Dies zeigt schon, dass das Interesse Goethes an der Anwendung wissenschaftlicher Metaphern auf die Literatur sehr wach ist. Man merkt übrigens aus ihren Briefen die Absicht, ihre literarischen und ästhetischen Werke zu verarbeiten. ${ }^{7}$ In diesem Sinne erweist sich der Briefwechsel in seinem Inhalt als klassisch, da er mancherorts «den Hintergrund des literarischen Schaffens bildet». ${ }^{8}$ Die chronologische Strukturierung des Briefwechsels, den wir hauptsächlich auf die Jahre zwischen 1798 und 1805 begrenzen - es sei denn Rückgriffe auf Briefe aus den Jahren 1794 bis 1797 erhellen das Ganze neu - begründet sich durch die Bedeutung, die der Übergang vom 18. zum 19. Jahrhundert darstellt, der in Deutschland den Übergang von der Aufklärung zur Romantik markiert und bedeutende Paradigmenwechsel mit sich gebracht hat.

\section{Der Briefwechsel als Ort intellektueller Anregung}

Gegenstand einer Perspektive auf die Briefe ist die Freundschaft zwischen den beiden 'Genies' ihrer Zeit. ${ }^{9}$ Generell lässt sich sagen, dass der Briefwechsel zwischen Goethe und Schiller weniger der Ort ist, in dem eine tiefe Freundschaft zum Ausdruck kommt und sich «die Stimme des Privatmannes hören lässt», wie Brigitte Diaz es formuliert hat (2002: 6), als der Ort einer fruchtbaren Koopera-

${ }^{7}$ Walter Hinderer: «Kreative Gegensätze. Zum ästhetischen Diskurs zwischen Goethe und Schiller», in: Der Briefwechsel zwischen Schiller und Goethe, op. cit., S. 69-84.

${ }^{8}$ Vom Französischen ins Deutsche von uns übersetzt.

${ }^{9}$ Darüber wird man den Beitrag von Ernst Osterkamp lesen können: «Wir. Was Goethe und Schiller unter Freundschaft verstehen", in: Der Briefwechsel zwischen Schiller und Goethe [hg. von Bernhard Fischer und Norbert Oellers], Erich Schmidt Verlag, 2011, S. 179 u. ff. 
tion. ${ }^{10}$ Die Freundschaft gründet ganz eindeutig auf intellektuellem Austausch. Sowohl Goethe als auch Schiller erhoffen sich von diesem beinahe alltäglichen Briefaustausch Impulse zur Arbeit (1984: 360), Ideen oder Anregungen (1984: 181-182) vielleicht sogar Komplimente (1984: 185) oder Bewunderung (1984: 207), was ihnen die nötige Energie einflößt, um an ihren Forschungen oder Kompositionen weiterzuarbeiten. Die folgende zitierte Textstelle scheint eindeutig zu zeigen, dass Goethe keinen Hehl daraus macht, dass er im Briefwechsel mit Schiller einen großen Vorteil sieht, wenn er am 6. Januar 1798 schreibt:

Das günstige Zusammentreffen unserer beiden Naturen hat uns schon manchen Vorteil verschafft, und ich hoffe, dieses Verhältnis wird immer gleich fortwirken [...] Sie haben mich die Vielseitigkeit des innern Menschen mit mehr Billigkeit anzuschauen gelehrt; Sie haben mir eine zweite Jugend verschafft und mich wieder zum Dichter gemacht, welches zu sein ich so gut als aufgehört hatte. (Goethe 1984: 11)

Die Briefe geben auch manchmal Zeugnis von ihrer performativen Funktion, d.h. dass sie vom Schreiben eine Wirkung auf die Realität intendieren, wie es folgende Passage aus einem Brief Goethes an Schiller zeigt: «[...] ich fühle ein wahres Bedürfnis, das Farbenwesen endlich einmal loszuwerden.» [...] «Was mich betrifft, so komme ich diesmal mit dem festen Vorsatz zu Ihnen, mir das Farbenwesen, es koste, was es wolle, vom Halse zu schaffen.» (Goethe 1984: 162 et 165)

\section{Dichtung und Philosophie. Dichtung und Wissenschaften}

Die thematische Kohärenz der Briefe Schillers fällt auf; Schillers Briefe kennzeichnet übrigens die Absicht, Goethe bei einer erneut intensiveren Auseinandersetzung mit der Poesie zu helfen. Dieser Aspekt soll im Folgenden im Mittelpunkt stehen, denn einer der wesentlichen Aspekte im Inhalt des Briefwechsels gilt für Schiller der Artikulierung zwischen Poesie und Philosophie und für Goethe dem geringeren Platz, den die Poesie nun in seinem Schaffen einnimmt, da er sich zu anderen Wissens- , Denkens- , und Schaffensbereichen hingezogen fühlt. Im ersten Teil des Briefwechsels erwähnt Goethe z.B. die von ihm geführten verschiedenen Forschungen auf dem Gebiet der Naturwissenschaften, der Metamorphose der Insekten, der Mineralogie, der Optik und sogar der Architektur.

Zu Beginn des Jahres 1798 kommt er noch einmal auf jene persönliche Problematik zu sprechen, die ihn ständig beschäftigt und die den gesamten Briefwechsel von 1798 bis 1805 durchzieht, d.h. die Abkehr von der Poesie - und der langsame Rückzug der Poesie in sein eigenes Schaffen (Goethe 1984: 340) - zu Gunsten der Philosophie: «Die Philosophie wird mir deshalb immer werter, weil sie mich täglich immer mehr lehrt, mich von mir selbst zu scheiden.» (Goethe 1984: 45)

${ }^{10}$ Lesley Sharpe: «Goethes und Schillers Theaterpartnerschaft. Ästhetik und Praxis im Spiegel ihres Briefwechsels», in: Der Briefwechsel zwischen Schiller und Goethe, ibid., S. 149-162. 
«Die philosophischen Kolloquia werden immer interessanter, und ich kann hoffen, wenn ich mir nur Zeit lasse, das Ganze recht gut einzusehen.» (Goethe 1984: 331).

Im Laufe der zehn Jahre seines Briefwechsels mit Schiller scheint Goethe, dessen Interesse für die Wissenschaften historisch belegt worden ist, sich übrigens für die Entwicklung der Wissenschaften seiner Zeit leidenschaftlich zu interessieren, was ihn von rein literarischen oder ästhetischen Reflexionen entfernt. Sein problematisches Verhältnis zur Dichtung muss ihm bewusst gewesen sein, wie folgender Textstelle zu entnehmen ist: «Wenn ich übrigens mit Niethammer und Friedrich Schlegel transzendentalen Idealism, mit Rittern höhere Physik spreche, so können Sie denken, dass die Poesie sich beinahe verdrängt sieht; doch läßt sich hoffen, dass sie wieder zurückkehren werde.» (Goethe 1984: 335). Er ist vertieft in die Redaktion seiner Farbentheorie, von der im Briefwechsel mehrmals die Rede ist $^{11}$ und an einigen Bemerkungen Schillers versteht man, dass die Arbeit an der Farbenlehre Goethe sehr lange beschäftigte: «Ihre lange Arbeit mit den Farben und der Ernst, den Sie darauf verwendet, muss mit einem nicht gemeinen Erfolg belohnt werden.» (Schiller 1984: 170) Während Schiller die Dichtung und die Philosophie in den Vordergrund seiner Ausführungen stellt, beschäftigt Goethe sich dagegen intensiv mit Wissenschaftsfragen. Folgerichtig entschließt er sich nicht mehr zu einer intensiven Arbeit an literarischen Werken. Die Orientierung seiner Überlegungen aus diesen Jahren ist also im Gegensatz zu den Positionen Schillers eine völlig andere.

Der intellektuelle Austausch zwischen Goethe und Schiller zeugt somit von einem Nicht-Parallelismus, von einer Asymmetrie in ihren jeweiligen Beschäftigungen: das Interesse Schillers gilt der Literatur und der Ästhetik - oder wenn er sich für andere Wissensgebiete interessiert, so ist es, weil sie möglicherweise der Literatur und der Ästhetik dienen können (Schiller 1984: 325) - während Goethe sein immer regeres Interesse für die Entwicklung der Wissenschaften und die wissenschaftlichen Experimente keineswegs verbirgt. Goethe gewinnt seine Einsichten über die Wissenschaft seiner Zeit durch Begegnungen mit markanten Wissenschaftlern wie mit dem Physiker Ritter oder dem Naturwissenschaftler Johann Friedrich Blumenbach. In einem Brief an Schiller vom 12. Juli 1801 teilt Goethe ihm sein Interesse für «die Schädensammlung» Blumenbachs mit und berichtet auch von einem Treffen mit dem Professor Hoffmann, der ihm dabei helfen soll, seine Kenntnisse auf dem Gebiet der Botanik zu erweitern: «Professor Hoffmann wird mich mit den kryptogamischen Gewächsen näher bekannt machen und dadurch eine starke Lücke in meinen botanischen Kenntnissen ausfüllen.» (Goethe 1984: 378). Goethe erzählt Schiller auch von seinen Mondbetrachtungen mit dem Teleskop in einem Brief vom 10. August und vom 21. August 1799:

Durch das Steinische Spiegelteleskop habe ich einen Besuch im Monde gemacht. [...] Diese Woche bin ich wider meine Gewohnheit meist bis Mitternacht aufgeblieben, um den Mond zu

${ }^{11}$ Darüber wird man die Briefe Goethes an Schiller vom 17. und 20. Januar, vom 31. Oktober und vom 7. November lesen. 
erwarten, den ich durch das Auchische Teleskop mit vielem Interesse betrachte. Es ist seine sehr angenehme Empfindung, einen so bedeutenden Gegenstand, von dem man vor kurzer Zeit so gut als gar nichts gewußt, um soviel näher und genauer kennenzulernen. (Goethe 1984: 255 et 262)

Am 14. Februar 1798 schreibt Goethe an Schiller, als wollte er selbst dazu beitragen, die Verspätung der Wissenschaften aufzuholen: «Dabei ist unglaublich, wie sehr die Wissenschaft retardiert worden ist.» (Goethe 1984: 48) Auf die Spitze getrieben ließe sich beinahe formulieren, dass Goethe die Retardierung der Wissenschaft aufholen möchte. In einem Brief an Goethe vom 23. Januar 1798 scheint Schiller sogar auf eine allgemeine Geschichte der Wissenschaften und des menschlichen Denkens anzuspielen, die Goethe vielleicht zu schreiben trachtete. «Das kleine Schema zu einer Geschichte der Optik enthält viele bedeutende Grundzüge einer allgemeinen Geschichte der Wissenschaft und des menschlichen Denkens, und wenn Sie sie ausführen sollten, so müssten sich viele philosophische Bemerkungen machen lassen.» (Schiller 1984: 26) Die Briefe Goethes verraten einen großen Gefallen an den jüngeren wissenschaftlichen Entdeckungen seiner Zeit; demgegenüber reagiert Schiller mit einer Mischung aus Ernst, Respekt und Bewunderung:

An Ihren Mondbetrachtungen wünschte ich wohl auch teilzunehmen. Mir hat dieser Gegenstand immer einen gewissen Respekt abgenötigt und mich nie ohne eine sehr ernste Stimmung entlassen. Bei einem guten Teleskop wird das Körperliche der Oberfläche sehr deutlich, und es hatte mir immer etwas Furchtbarer, dass ich diesen entfernten Fremdling auch mit einem andern Sinn als dem Aug zu erfassen glaubte. (Schiller 1984: 262)

Zwar scheint Schiller sich dafür zu interessieren und Neugier zu zeigen, aber er ist auch darüber besorgt, dass das große Interesse Goethes für das wissenschaftliche Gebiet die Entfaltung seines poetischen Genies bremst oder gar verhindert. So schreibt er ihm am 16. Juli 1798: «Ich bin begierig über Ihre mit den großen eisernen Massen und dem Magnet neu gemachten Entdeckungen. Wenn Ihnen das nächste Vierteljahr notwendig so zerstückelt werden soll, so wird das Poetische freilich zu kurz kommen, dafür aber können Sie in diesen physischen Dingen desto weiter kommen, welches auch nicht schlimm ist.» (Schiller 1984: 115) Schiller befürchtet, dass das tiefe Interesse seines Freundes für die neueren wissenschaftlichen Entdeckungen oder die neuen Maß - oder Optikinstrumente ihn von dem wahren Ausdruck seines Genies, d.h. der Poesie ablenkt:

Ich freue mich auf den magnetischen Kursus sehr; in dem Fischerischen Wörterbuch habe ich gerade über diesen Gegenstand wenig Trost gefunden, da dieser erste Band nicht so weit reicht. Wir wollen dann auch, wenn es Sie nicht zerstreut ${ }^{12}$, über Elektrizität, Galvanism und chemische Dinge uns unterhalten und wo möglich Versuche anstellen. (Schiller 1984: 109)

Ein Jahr später, am 5. März 1799, wird sein Ton schon entschiedener und er weist sogar Goethe zurecht: «Eine so lange Pause, als Sie damals in der Poesie gemacht haben, darf nicht mehr vorkommen, und Sie müssen darin ein Machtwort

12 Hervorhebung von uns. 
aussprechen und ernstlich wollen.» (Schiller 1984: 198) Zwei Jahre später erfährt er sehr erleichtert, dass Goethe wiederum sowohl in Harmonie mit den Musen zusammenlebt als auch von den Wissenschaften und der Philosophie Abstand hält. «Dass Sie unterdessen mit den Musen allein leben und die Philosophen verbannt haben, hören wir mit großem Vergnügen.» (Schiller 1984: 344) Schiller sieht dennoch in Goethe einen zukünftigen Wissenschaftler und ermutigt ihn sogar, seinem wissenschaftlichen Schaffen eine didaktische Orientierung zu geben. In einem Brief an Goethe vom 30. November 1798 schreibt er: «Sie müssen, da Sie es können, ein Muster aufstellen, wie man physikalische Forschungen behandeln soll, und das Werk muss durch seine Behandlung ebenso belehrend sein als durch seine Ausbeute für die Wissenschaft. [...] so ist ein unsterblicher Name in der Wissenschaft etwas sehr Wünschenswürdiges.» (Schiller 1984: 170)

Genauere Hinsicht zeigt, dass politische Betrachtungen in dem Briefwechsel zwischen Goethe und Schiller entweder völlig abwesend sind oder nur kurz und oberflächlich erwähnt werden. (Schiller 1984: 29) Der Genauigkeit der Informationen über den Stand der Literatur oder der Philosophie steht das Fehlen jeder Mitteilung über politische Ereignisse entgegen. In diesem Sinne scheinen Goethe und Schiller wohl, im Licht ihres Briefwechsels, die Vertreter einer Epoche zu sein, die ihre Schwäche auf dem politischen Gebiet durch Stärken in den Bereichen der Wissenschaften und der Literatur auszugleichen sucht. ${ }^{13}$ Aber interessant in diesem Briefwechsel ist eben die Idee einer möglichen Versöhnung zwischen den Künsten und den Wissenschaften, der Wissenschaft und der Literatur, der Poesie und der Philosophie, der Praxis und der Theorie (Goethe 1984: 431); viele der zwischen Goethe und Schiller ausgetauschten Briefe zeugen in dieser Hinsicht von einer Kritik des Dualismus und der Notwendigkeit ihn zu überschreiten.

\section{Brief versus Gespräch}

Mit den Worten "Brief" und "Gespräch" sind die beiden Stichworte gefallen, die nun im Mittelpunkt der Aufmerksamkeit stehen und vorliegender Abschnitt setzt sich mit dem Charakter des Briefes als Gattung auseinander. ${ }^{14}$ An manchen Textstellen scheint sich ein Gattungsproblem aufzutun, das filigranartig von beiden Schriftstellern diskutiert wird. Die Korrespondenz zwischen Goethe und Schiller ist auch insofern interessant, als die beiden Schriftsteller mancherorts sich über die

${ }^{13}$ In einem Brief an Goethe vom 21. Dezember 1803 betont Schiller, indem er auf Madame de Staël zurückgreift, dass die Deutschen ihre nationale Identität dank der Poesie und der Philosophie behaupten werden, im Gegenteil zu ihren Nachbarn, den Franzosen, die Madame de Staël zufolge, keinen Sinn für Poesie besitzen.

14 Über die manchmal verschwommene Grenze zwischen Brief und Essay kann man den Beitrag von Wilfried Barner lesen: «Brief oder Essay? Gedankenexperimente in Schillers und Goethes Korrespondenz», in: Der Briefwechsel zwischen Schiller und Goethe, op. cit., S. 35-52. 
Gattung des Briefes ${ }^{15}$ selbst austauschen, deren Grenzen umrissen werden müssen im Hinblick auf das Gespräch, das eine von den Intellektuellen dieser Zeit hochgeschätzte andere Gattung darstellt. Die Verwandschaft zwischen dem Brief und dem Gespräch ist so eng, dass Brigitte Diaz den Brief sogar als «die schriftliche Übertragung eines Gesprächs, das nicht stattgefunden hätte» (Diaz 2002: 24), als «ein Notbehelf des Gesprächs» (Diaz 2002: 25) definiert. ${ }^{16}$ Auf Grund seines formalen und rhetorischen Rahmens macht der Brief die Ausführungen nicht immer möglich, die die beiden Schriftsteller und Denker sich wünschen, als wäre einzig das Wort dazu fähig, ihnen in ihrer Reflexion weiterzuhelfen. So schreibt Goethe am 6. Januar 1798 an Schiller: «Bei Gelegenheit des Schellingischen Buches habe ich auch wieder verschiedene Gedanken gehabt, über die wir umständlicher sprechen müssen.» (Goethe 1984: 13) Schiller seinerseits erklärt sich mit der Idee, dass der Brief als Gespräch gelten kann (Diaz 2002: 115), nicht einverstanden und beharrt in einem Brief vom 19. Januar 1798 auf der großen Bedeutung des Gesprächs, das ihn einzig in intellektueller Hinsicht unterstützen kann: «Denn nur das Gespräch hilft mir eigentlich [...] In dem Monolog eines Briefes ${ }^{17}$ bin ich stets in Gefahr, nur meine Seite zu fassen.» (Schiller 1984: 24) Noch ausgeprägter erscheint eine weitere Passage, die einem Brief an Goethe vom 6. März 1798 entnommen ist und von der großen Bedeutung zeugt, die Schiller dem Gespräch als dynamische Unterstützung für das Denken und die Reflexion beimisst: «Ihre vielen und reichen Erfahrungen und Reflexionen über Natur und Kunst und über das dritte Idealische, was beide zuletzt zusammenknüpft, müssen ausgesprochen ${ }^{18}$, geordnet und festgehalten werden.» (Schiller 1984: 66-67) oder auch «Die neulichen Unterhaltungen mit Ihnen haben meine ganze Ideenmasse in Bewegung gebracht.» (Schiller 1950: 13) Am 29. September desselben Jahres beweist Goethe, dass das Gespräch einen größeren sozialen als intellektuellen Vorteil hat, wenn er an Schiller schreibt: «Bei dem trüben Himmel ist das Gespräch noch der einzige Trost.» (Goethe 1984: 147)

Im Gegensatz zu Schiller scheint Goethe dem Brief eine größere intellektuelle Bedeutung als dem Gespräch beizumessen (Goethe 1950: 30). So schreibt er am 20. Juli 1799 an seinen Freund bezüglich der neueren Veröffentlichung des Romans Friedrich Schlegels Lucinde, der einen großen Skandal hervorrief: «Ich

15 Die Anführungszeichen sollen hier die Schwierigkeit hervorheben, auf die man stößt, wenn es darum geht, den Brief einer klaren Gattung zuzuordnen. Wie Brigitte Diaz es erklärt, «sind Briefwechsel Hybridtexte, die Gattungszugehörigkeiten herausfordern. Als unauffindbare literarische Gattung schweben Briefwechsel zwischen unbestimmten Kategorien: Archiv, Dokumenten, Zeugenaussagen.»

16 Übersetzung - BA.

17 Trotz seiner sogenannten dialogischen Struktur hat der Brief also auch eine selbstreflexive Dimension.

${ }^{18}$ Hervorhebung von uns. 
danke Ihnen, dass Sie mir von der wunderlichen Schlegelischen Produktion einen nähern Begriff geben, ich hörte schon viel darüber reden.» (Goethe 1984: 241)

In einem Brief von Schiller an Goethe vom 23. Juni 1799 liest man, wie der Dialog für Schiller eine klärende Funktion besitzt, was ihn besonders wertvoll macht: «Ich habe heute Ritters Schrift über den Galvanism in die Hand bekommen, aber obgleich viel Gutes darin ist, so hat mich die schwerfällige Art des Vortrags doch nicht befriedigt und auf eine Unterhaltung mit Ihnen über diese Materie nur desto begieriger gemacht.» (Schiller 1984: 121-122) Er empfindet den literarischen Raum des Briefes als zu begrenzt und seine Briefe können zu wahren Abhandlungen werden, so sehr er sich über manche Themen wie das dichterische Schaffen oder die Kritik auslassen kann. Er schreibt beispielsweise an Goethe am 23. August 1794: «Aber ich bemerke, dass ich anstatt eines Briefes eine Abhandlung zu schreiben im Begriff bin.» (Schiller 1950: 15)

Der Brief bildet meist eine Art Prolog zu einem Gespräch, von dem Goethe und Schiller sich eine Vertiefung ihres schriftlichen Austauschs erhoffen. Nicht selten legen sie ihren Briefen ein kleines Paket mit einem Buch oder einem Manuskript bei, das dazu bestimmt ist, ein zukünftiges Gespräch zu nähren. Beispielsweise begleitet Goethe einen Brief an Schiller vom 10. Januar $1798 \mathrm{mit}$ den Ästhetischen Betrachtungen Bouterweks. «Zugleich lege ich des Herrn Bouterweks ästhetische Bemühungen bei.» (Goethe 1984: 15) Zwei Tage später bekommt er folgende Antwort von Schiller, der sich inzwischen in die Lektüre des Werks Bouterweks vertieft hat und sich heftig über den sogenannten «Bouterweks ästhetische[n] Kramladen» äußert. «Bouterweks ästhetischer Kramladen ist wirklich merkwürdig. Nie hab' ich den flachen belletristischen Schwätzer mit dem konfusen Kopf so gepaart gesehen und eine so unverschämte Anmaßung auf Wissenschaft bei einem so erbärmlich rhapsodistischen Hausrat.» (Schiller 1984: 17)

Wenn sie mit dem Urteil über Bouterwek übereinstimmen, haben ihre Bemerkungen darüber doch einen Unterschied in ihrem jeweiligen Charakter. Goethe entpuppt sich in seinen Äußerungen als viel umsichtiger und zurückhaltender als Schiller. Den «ästhetischen Bemühungen Bouterweks» bei Goethe entspricht bei Schiller «Bouterweks ästhetischer Kramladen»; dort wo Goethe über «unbestimmte Standpunkte» spricht, betrachtet Schiller das Gelesene als eintönig, konfus, schamhaft, ja beinahe peinlich. Durch solche Äußerungen beweisen sie, dass ihre Begeisterung und ihre Energie nicht den gleichen Schaffenspolen gelten. Während Schiller sich eher zurückhaltend zeigt, wenn es darum geht, ein Urteil über die Schriften des Physikers Ritter zu fällen, ist er dagegen besonders kritisch, wenn er über ein ästhetisches oder literarisches Werk urteilen soll. 


\section{Der Brief als Raum der Kritik und Labor des Schaffens}

Der Brief wird somit zum Raum einer kritischen Untersuchung der jüngsten Veröffentlichungen oder der intellektuellen Tendenzen ihrer Zeit. Die Kommentare Goethes bezüglich der Schriften Ritters zeugen von seiner wissenschaftlichen Schärfe und Genauigkeit sowie von seinem Willen, zur Entstehung einer auf der Vernunft begründeten Wissenschaft beizutragen, die von Vorurteilen oder strengen Urteilen befreit wäre. In dieser Hinsicht kritisiert er scharf die Schriften Schlossers, die Schiller übrigens in einem Brief vom 9. Februar der Kategorie der sogenannten «Unphilosophen» schon zugeordnet hatte. Als Antwort auf diesen Brief antwortet Goethe ihm am 13. Februar 1798, wobei er ihn in seiner Überzeugung stärkt: «die Schlosserische Schrift [...] da ich eben in einem wissenschaftlichen Fache in dem Falle bin, über beschränkte Vorstellungsarten, Starrsinn, Selbstbetrug und Unredlichkeit zu denken, so war mir diese Schrift ein merkwürdiger Beleg.» (Goethe 1984: 44) Noch strenger wirkt ihre gemeinsame Einschätzung der Gebrüder Schlegel, die sie sogar für verrückt halten. In einem Brief an Schiller vom 28. Juni 1798 schreibt Goethe: «Desto entschiedner ist der Brief, den Sie zugleich erhalten, und ein herrliches Muster einer Tollheit außer dem Tollhause. Denn das Kriterium, warum man einen solchen Menschen nicht einsperrt? möchte schwer anzugeben sein. [...] Da ich ihn aber nicht einsperren kann, so soll er wenigstens ausgesperrt werden.» (Goethe 1984: 108)

In mehreren Passagen ihres Briefwechsels ist auch eine scharfe Kritik am Rationalismus zu lesen; sie bildet z.B. den Kern eines Briefes, den Schiller am 19. Januar 1798 an Goethe schreibt. Der Rationalismus trennt das, was zusammengehört und trägt somit dazu bei, eine dualistische Weltanschauung zu erhalten, die in der zweiten Hälfte des 18. und zu Beginn des 19. Jahrhunderts eben Objekt zahlreicher Kritiken geworden ist.

Die Verbindung der Sinne und der Vernunft wird in einem Brief von Schiller an Goethe vom 19. Januar 1798 herauf beschwört:

[...] so finden wird auch, dass nur die vollkommene Wirksamkeit der freien Denkkräfte mit der reinsten und ausgebreitetsten Wirksamkeit der sinnlichen Wahrnehmungsvermögen zu einer wissenschaftlichen Erkenntnis führt. (Schiller 1984: 23) Er kommt in Gefahr, dasjenige strenge zu sondern, was in der Natur verbunden ist, wie er oben verband, was die Natur scheidet. Er macht Einteilungen, wo keine sind [...] das ewige Bestreben des Rationalism, nach der Kausalität der Erscheinungen zu fragen und alles qua Ursach und Wirkung zu verbinden. (Schiller 1984: 21)

In einem Brief vom 20. Januar 1798 (1984: 25) erwähnt Goethe, nicht ohne eine gewisse Ironie, «den nach Ursachen haschenden Rationalism». Dem rationalistischen Denken wirft er sein einseitiges Funktionieren vor, sowie die Art und Weise, wie er ausschließlich einen einzigen Aspekt der Natur in Betracht zieht: «Der Rationalism scheint hier vorzüglich dadurch zu fehlen, dass 
er dürftigerweise bloß die Länge und nicht die Breite der Natur in Anschlag bringt.» (Goethe 1984: 21) Das ist auch eine von Goethe formulierte Kritik, wie Schiller es in demselben Brief erwähnt: «Ich beziehe mich hier auf Ihren Aufsatz selbst, der vorzüglich diesen Mißbrauch, den die Kausalbestimmung der Phänomene veranlaßt, rügt.» (Schiller 1984: 21) Was Schiller sich wünscht, ist der rationalistische Empirismus und dessen Triumph über die reine Theorie: «Zu dem reinen Phänomen, welches nach meinem Urteil eins ist mit dem objektiven Naturgesetz, kann nur der rationelle Empirism hindurchdringen.» (Schiller 1984: 22)

Mehrere der zwischen den beiden Männern ausgetauschten Briefe enthalten außerdem Betrachtungen über ihre Arbeitsweise und die Schwierigkeiten, auf die sie beim Vorantreiben ihrer wissenschaftlichen oder literarischen Arbeiten stoßen. Indem sie mit ähnlichen Bemerkungen den Leser hinter die Kulissen ihrer jeweiligen Schaffenswerke führen, verändern sie auch den Blick, den man auf ihr sogenanntes schöpferisches Genie werfen kann. Wenn man einige Worte liest, die die beiden «Riesen» des deutschen Denkens austauschen, ist das schöpferische Genie ohne eine Menge Arbeit nicht denkbar. Goethe schreibt an Schiller am 10. Januar 1798 (1984: 15): «Denn selbst jetzt, da ich mich so weit durchgearbeitet habe, bedarf es noch einer großen Arbeit, bis ich mein Material zu einer reinen Darstellung bringe.» Zwei Monate später, am 6. März 1798 (1984: 67), ermutigt Schiller Goethe zum Schaffen, wobei er dabei auf die Arbeitsspirale hinweist, in die Goethe hineingeraten wird: «Aber die Unternehmung wird weitläufig werden, und aus Arbeit wird sich Arbeit erzeugen.» Wenig später, am 18. Juli 1798 (1984: 116), teilt Goethe Schiller mit, dass er bei der Verfassung mancher Werke auf große Schwierigkeiten stößt: «Die Hauptschwierigkeit bei der Redaktion ist vom Anfang, dass man die allgemeinen Zwecke immer im Auge habe und bei allen fragmentarischen Wesen auf ein Ganzes hindenke», was er auch in einem späteren Brief an Schiller vom 6. Oktober bestätigt (1984: 155): «Ich arbeite nur, dass alles Einzelne herausgehoben werde und sich ins Ganze anschließe.»

Der schriftliche Raum des Briefwechsels wird auch manchmal zu einem literarischen Ort, wo methodologische Ratschläge ausgetauscht werden können. Goethe schreibt z.B. am 14. August 1799 an Schiller (1984: 257): «Lassen Sie es ja an Konzentration auf Ihre angefangene Arbeit nicht fehlen. Es ist doch im Grunde nichts wünschenswerter, als eine große Masse zu organisieren.» In einem Brief an Goethe vom 27. März 1801 erklärt Schiller (1984: 365), was den Auftakt zu einer Schöpfung bilden soll, d.h. eine dunkle, aber mächtige Totalidee: «Ohne eine solche dunkle, aber mächtige Totalidee, die allem Technischem vorhergeht, kann kein poetisches Werk entstehen. [...] Aus der Idee aber kann ohne die Tat gar nichts werden.» 


\section{Ein mit seltenen sozialen oder politischen Betrachtungen durchzogener Korpus}

Abschließend soll skizziert werden, dass Goethe und Schiller durch den kritischen und distanzierten Blick, den sie sowohl auf Europa als auch auf ihre englischen und französischen Nachbarn werfen, Intellektuelle ihrer Zeit sind, insofern als sie auf eine auf Europa zentrierte oder auf Deutschland reduzierte Anschauung verzichten. Nichtsdestotrotz sind Spuren nationalen Stolzes in vereinzelt auftretenden Bemerkungen über den Zustand der französischen oder englischen Literatur spürbar, die an den Willen der Deutschen erinnert, sich statt auf dem politischen, auf dem literarischen Gebiet durchzusetzen: «Die französische Sprache ist aber auch recht dazu gemacht, um die Erscheinung der Erscheinungen auszudrücken, übrigens scheinen ihre Literatoren so zahm, als ihre Politik gewaltsam ist.» schreibt Goethe an Schiller am 14. März 1798 (1984: 73). Es ist eine der seltenen von ihm zur Politik überlieferte Stellungnahme. Sie sind auch Intellektuelle ihrer Zeit in dem Sinne, als sie ein kritisches Urteil fällen und einen distanzierten Standpunkt haben, wenn sie sich über ihre Zeitgenossen und Landsleute äußern. Schiller schreibt am 30. Januar an Goethe (1984: 34): «Die Schrift von Darwin würde wohl in Deutschland wenig Glück machen. Die Deutschen wollen Empfindungen, und je platter diese sind, desto allgemeiner willkommen.» In einem Brief, den Goethe ihm am nächsten Tag schickt, setzt er die Diskussion fort und erwähnt den Mangel an Humor der Deutschen, die wegen ihrer Philisterhaftigkeit dem Humor nicht zugänglich sind: «Dafür hat der Deutsche so selten Sinn, weil ihn seine Philisterhaftigkeit jede Albernheit nur ästimieren lässt, die einen Schein von Empfindung oder Menschenverstand vor sich trägt.» (1984: 36)

Abschließend kann man sagen, dass der Briefwechsel zwischen Goethe und Schiller, aus dem wir absichtlich nur die Jahre von 1798 bis 1805 in Betracht gezogen haben, eine ziemlich unsymmetrische Beziehung wiedergibt. Im Grunde genommen handelt es sich weniger um einen Briefwechsel zwischen Schriftstellern als um einen brieflichen Austausch, der durch eine stark spürbare Spannung zwischen einem erfahrenen und begeisterten Schriftsteller und einem Denker gekennzeichnet ist, der all diese Jahre mit Problemen wissenschaftlicher und philosophischer Natur beschäftigt ist, die ihn provisorisch von der Dichtung ablenken und ihn dazu führen werden, die Literatur anders zu betrachten. Goethe entwirft übrigens im Laufe dieses Briefwechsels ein Selbstporträt, das seine intellektuellen Interessen oder Geschmäcker als sehr eklektisch erscheinen lässt und die Briefe zu einem Raum für sprachliche Identitätsentwürfe und rhetorische Stilisierung macht. ${ }^{19}$ Er beschreibt sich selbst bald als absoluter Realist, bald als perfekter

19 Über formale und stilistische Aspekte der Korrespondenz, siehe: Alice Staskova: «Stil und Rhetorik im Briefwechsel zwischen Schiller und Goethe», in: Der Briefwechsel zwischen Schiller und Goethe, op. cit., S. 53-68. 
Idealist (1984: 83), schließlich als praktischer Skeptiker (1984: 93) oder als Naturbeobachter (1984: 109). In Anbetracht der für ihre rein literarischen Austausche ${ }^{20}$ bedrohlichen intellektuellen Zersplitterung seines Freundes, trachtet Schiller nach einem Kompromiss und einer Versöhnung der Interessenpole Goethes. Filigranartig schlägt er vor, über den Dualismus zwischen Literatur und Wissenschaft hinauszugehen, d.h. die Begrenzungen des Dualismus zu durchbrechen, um zu der Möglichkeit eines wissenschaftlichen Diskurses über Kunst und Literatur zu kommen, wodurch Goethe sein dichterisches Genie und seine wissenschaftlichen Arbeiten verbinden, bzw. versöhnen könnte:

Schon deswegen ist mir Ihre Idee zu einem didaktischen Gedichte sehr willkommen gewesen; eine solche Beschäftigung knüpft die wissenschaftlichen Arbeiten an die poetischen Kräfte an und wird Ihnen den Übergang erleichtern, an dem es jetzt allein zu fehlen scheint. (Schiller 1984: 198) [...] nun möchte noch etwas Allgemeines und, wenn Sie wollen, Szientifisches über das eigentlich Künstlerische zu sagen sein. (Schiller 1984: 338)

Anhand des vorausgehenden Zitats lässt sich zeigen, dass in der deutschen Gedankenwelt um 1800 zwei intellektuelle Tendenzen miteinander konkurrieren; der Briefwechsel gibt vor allem Zeugnis von einer nach Überschreitung des Dualismus fragenden Perspektive.

\section{Literatur}

\section{Quellen}

Johann Wolfgang Goethe mit Schiller: Briefe, Tagebücher und Gespräche vom 24 Juni 1794 bis zum 9 Mai 1805, Deutscher Klassiker Verlag, Bde 4 et 5, Frankfurt am Main 1998 [zit. nach: Johann Wolfgang Goethe, Briefwechsel mit Friedrich Schiller, Artemis-Verlag, Zürich 1950 und Der Briefwechsel zwischen Schiller und Goethe. Briefe der Jahre 1798-1805, Zweiter Bd. Insel Verlag, Leipzig 1984].

\section{Sekundärliteratur}

\section{Monographien}

Boemer, Peter: Johann Wolfgang von Goethe. (= Rowohlt Monographien), Hamburg 1990.

Diaz, Brigitte: L'épistolaire ou la pensée nomade. Paris 2002.

Friedenthal, Richard: Goethe. Sein Leben und seine Zeit. (Band II), München 1968.

Lukács, Georg: Goethe und seine Zeit. Berlin 1955.

Safranski, Rüdiger: Goethe und Schiller: Geschichte einer Freundschaft. München 2009.

${ }^{20}$ Darüber kann man z.B. in dem Brief Goethes an Schiller vom 17. August 1796 lesen, in dem er schreibt: «Da ich den Roman los bin, so habe ich schon wieder zu tausend andern Dingen Lust.», ibid., S. 243. 


\title{
Sammelarbeiten
}

Fischer, Bernhard / Oellers, Norbert (Hrgs.): Der Briefwechsel zwischen Schiller und Goethe. Berlin 2011.

Henke, Silke / Rosenbaum Alexander (Hrgs.): Zweiheit im Einklang: der Briefwechsel zwischen Schiller und Goethe; eine Ausstellung des Goethe-und Schiller-Archivs im Renaissancesaal der Herzogin Anna Amalia Bibliothek, 18.09.2009 bis 17.01.2010, Weimar 2009.

\begin{abstract}
s
Aus der Begegnung zwischen Goethe und Schiller im Juli 1794 in Jena wird eine tiefe Freundschaft, die sich durch einen intensiven Briefwechsel bis zum frühzeitigen Tod Schillers im Jahre 1805 nähren wird. Dieser formal klassische Briefwechsel, der dem Brief öfters einen kritischen Freiraum verleiht oder ihm die Funktion eines Labors des künstlerischen oder literarischen Schaffens gibt, zeichnet sich durch Originalität am Übergang vom 18. zum 19. Jahrhundert aus, übt Kritik am Dualismus und öffnet die Perspektiven hinsichtlich einer Versöhnung zwischen bis dahin getrennten Denkgebieten. Die Originalität in den Briefen zeigt sich am Vorschlag, die Wissenschaften und die Literatur nicht mehr einander entgegenzustellen, sondern sie zu artikulieren und somit die Möglichkeit eines wissenschaftlichen Diskurses über das literarische Werk in Betracht zu ziehen.
\end{abstract}

Schlüsselwörter: Dichtung, Philosophie, Kritik, Wissenschaften, Gespräch

\section{Progressive emerging scientific discourse on arts and literature around 1800 in light of the correspondence between Goethe and Schiller (1798-1805)}

Following their meeting in Iena in July 1794, Goethe and Schiller developed a strong friendship and kept up intense correspondence until Schiller's premature death in 1805. This exchange of letters opens up the space for a critical debate and confers upon the letter the status of a laboratory for artistic and literary creation. The correspondence also encodes one of the more original aspects of the late 18th- to early 19th-century thought: a critique of dualism which leads to the perspective of a reconciliation between science and literature, fields of thought hitherto kept separate. Articulating rather than opposing these fields, Goethe and Schiller conceive of the possibility of holding a scientific discourse on literature.

Keywords: poetry, philosophy, criticism, sciences, conversation

\author{
Bénédicte Abraham \\ Franche-Comté (Besançon) \\ Institut für Germanistik \\ 30, rue Mégevand \\ 25030 Besançon cedex \\ Frankreich \\ E-Mail: benedicte.abraham@univ-fcomte.fr
}

